\title{
Mental layouts of concealed objects as a function of imagery type and experimental conditions
}

\author{
JAMES IACCINO and JAMES BYRNE \\ Illinois Benedictine College, Lisle, Illinois
}

(Brian H. Ross, Sponsor)

\begin{abstract}
We examined whether concealed imagery was an effective mnemonic aid in the immediate recall of paired objects by conducting a study that contained explicitly worded instructions to conceal certain objects. Forty subjects were presented with counterbalanced verbal descriptions of concealed, pictorial, and separate object interactions; the descriptions were further categorized according to the type of image elicited, whether normal or bizarre. Results replicated Kerr and Neisser's (1983) findings, with pictorial interactions being recalled better than were concealed or separate. Furthermore, the bizarre effects obtained suggested these types of images had poorer recall and elicited lower vividness ratings than did normal types across the three conditions, with bizarre concealed images generating the lowest ratings of all. It remains to be determined whether these images can be mnemonically effective over delayed recall periods.
\end{abstract}

The current view pertaining to concealed imagery (i.e., the placement of objects in nonvisible locations within mental layouts) is that subjects need special instructions to actually visualize scenes exactly as described to them. Otherwise, they might change the spatial arrangements of the objects, mentally "seeing and feeling" the concealed items as though they were truly visible (see Keenan, 1983; Kozminsky, 1981).

Studies have shown that when explicit experimental instructions were provided in the strongest wording possible to conceal elements, concealed images were remembered no better than were noninteracting separate ones, while visible pictorial images elicited the best recall (Keenan \& Moore, 1979; Kerr \& Neisser, 1983; Kozminsky, 1981; Zimler \& Keenan, 1983). Instructions apparently reduced or eliminated the visibility of concealed objects, making them less memorable than the pictorial ones. As reported by Keenan and Moore (1979), instructions also have affected the rated vividness of these items, with concealed images being viewed less clear than were pictorial types.

In all of these designs, the effects of bizarre imagery have not been controlled. Some sentence frames employed did not depict the typical, commonplace interaction between objects. For example, unusual pictorial and concealed frames used in Neisser and Kerr's studies (Kerr \& Neisser, 1983; Neisser \& Kerr, 1973) consisted of the following: "A harp is sitting on top of the torch held up by the Statue of Liberty" and "Buried beneath a snowbank, there is a clock." Even historical figures and cartoon characters (Zimler \& Keenan, 1983) were involved in some of the image scenes: "A revolver is inside the breast pocket of Napoleon's coat" or "There is a cement truck locked inside Charlie Brown's garage." Although

Correspondence may be sent to James Iaccino, Department of Psychology, Illinois Benedictine College, Lisle, IL 60532. some researchers (Wollen, Weber, \& Lowry, 1972) believe that recall performance is affected by the interaction between the elements of the image and not their bizarreness, the influence of this variable on concealed imagery per se has never been determined. Only under pictorial conditions has a consistent finding with this type of imagery been found, namely, that bizarre interacting pairs did not exert a significant effect on immediate recall of paired associates over more normal commonplace scenes (Cox \& Wollen, 1981; Hauck, Walsh, \& Kroll, 1976; Kroll, Schepeler, \& Angin, 1986; Nappe \& Wollen, 1973; Senter \& Hoffman, 1976; Wood, 1967). In fact, in some studies immediate retention was adversely affected by these bizarre elements (Collyer, Jonides, \& Bevan, 1972; Wollen \& Cox, 1981).

On the basis of these limited investigations with bizarreness, we hypothesized an interaction between image type and experimental conditions, with the pictorial conditions eliciting the best recall and highest clarity under both bizarre and normal imagery. However, the normal types would still be more effective than would the bizarre in terms of immediate recall and rated vividness. To ensure appropriate differentiation between bizarre and normal types of images, a procedural modification recently introduced by Iaccino and Spirek (1988) was used. Specifically, scenes depicting pictorial, concealed, or separate objects would be rated initially on their level of bizarreness, and those with the most reliable ratings would be employed in the main experiment.

\section{METHOD}

\section{Subjects}

Forty male and female students ( $M=20.7$ years) from Illinois Benedictine College at Lisle participated in the main study for credit toward a course requirement in psychology. They were not preselected for ability to image. 


\section{Materials}

Materials consisted of 80 high-imagery nouns, ranging from 6.20 to 6.80 on the imagery scale of Paivio, Yuille, and Madigan's (1968) word lists. The items selected were arranged to form $\mathbf{4 0}$ pairs of words that were described in sentences. The first sentence had the stimulus term of each pair, and the second sentence connected the stimulus to the response term. The second sentence also described the response term (or target object) as pictorial, concealed, or separate. The pictorial condition visibly related the target to the stimulus term in the initial sentence. The concealed version also related both terms, but the target object was obstructed or hidden from view. Finally, the separate version described the target as spatially set apart from the stimulus. Care was taken in positioning each target word as close to the end of the second sentence as possible without losing coherency.

Approximately 200 subjects were initially given the $\mathbf{4 0}$ descriptive pairs of sentences in all three versions and were asked to rate each version on a 5-point scale, extending from most normal (1) to most bizarre (5). Half of the subjects rated the bizarre versions, and the remaining members read the normal types and assigned values to these scenes. Examples of the sentence pairs are provided in Table 1.

Rank order coefficients of .65 and .73 were subsequently obtained on the subjects' averages for the normal and bizarre types of sentences, respectively, suggesting a very high degree of agreement within the groups. Mean ratings were 1.75 for the normal pairs $(S D=0.88)$ and 4.34 for the more bizarre pairs $(S D=1.42)$.

\section{Design}

From the $\mathbf{4 0}$ sentence pairs, 18 were selected and used in the main study. These pairs elicited the highest degree of consistency among raters, as evidenced by their most extreme values. Each of the $\mathbf{4 0}$ subjects tested received the initial sentence frame; however, only one version of the second sentence was provided. Six versions were descriptive of the pictorial condition, six descriptive of the concealed condition, and six descriptive of the separate. To prevent primacy and recency effects, three buffer pairs preceded and followed the 18 experimental sentences. These six buffers were not included in the recall test or in the data analysis.

The subjects were subdivided in terms of the sentences' image type: 20 were given normal descriptions and 20 received the bizarre scenes. The target words were presented in the same order for all subjects, and, on subsequent recall, the 18 scenes were provided in the same order. The 18 target responses to be remembered consisted of the following: ambulance, beaver, book, bullet, butterfly, candy, caterpillar, cigar, corn, doctor, gem, gold, hammer, lobster, plank, string, thorn, and wine. The type of design employed was a $2 \times 3$ factorial combination of im-

Table 1

Sample Sentence Conditions as a Function of Image Type

\begin{tabular}{ll}
\hline Image Type & \multicolumn{1}{c}{ Normal } \\
Basic Frame & $\begin{array}{l}\text { A huge avalanche of snow } \\
\text { rumbles down the mountain. } \\
\text { Directly in the path of the huge } \\
\text { avalanche lies a rustic cabin. } \\
\text { Buried under the tons of the } \\
\text { avalanche, there is a rustic cabin. } \\
\text { On the opposite mountainside away } \\
\text { from the avalanche sits a rustic cabin. }\end{array}$ \\
Separate & $\begin{array}{l}\text { Bizarre } \\
\text { Basic Frame }\end{array}$ \\
Pictorial & $\begin{array}{l}\text { A huge avalanche of snow } \\
\text { rumbles down the mountain. } \\
\text { And the avalanche is stopped in } \\
\text { its tracks by a rustic cabin. } \\
\text { Directly under the tons of avalanche }\end{array}$ \\
Concealed & $\begin{array}{l}\text { a rustic cabin cries for help. } \\
\text { And the avalanche tries to find a } \\
\text { rustic cabin lying far away. }\end{array}$
\end{tabular}

age type (normal vs. bizarre) and experimental conditions (pictorial, concealed, and separate), with conditions being a within-subjects factor

\section{Procedure}

The subjects were tested individually. They were told that they would hear a series of 24 narrated scenes and that they should try to imagine each scene exactly as described to them. The subjects were also informed to rate each image they formed on a 7-point scale, extending from perfectly clear and vivid (1) to completely vague and dim (7). The scale was a shortened form of the Bett's questionnaire on mental imagery adapted by Sheehan (1967).

Instructions to conceal particular target words were also provided: "Now, some of the narrated scenes will describe objects that are concealed. For example, an object might be inside, under, or behind another object. It is very important that you imagine the scenes exactly as I describe them to you. For example, if I read you the description, 'A huge avalanche of snow rumbles down the mountain. Buried beneath the tons of avalanche there is a rustic cabin,' you should imagine the scene exactly as described with the cabin beneath tons of snow on top. You should not be able to see the cabin at all. For any of these narrated scenes, do not try to see the concealed object."

These instructions were similar in format to those used by Kerr and Neisser (1983). Practice descriptions with all three conditions were given to ensure that the subjects understood the task.

The 24 descriptions immediately followed the practice conditions. The scenes were read one at a time, then an unexpected recall test was administered after a retention interval of approximately $3 \mathrm{~min}$. The experimenter gave each of the $\mathbf{1 8}$ stimulus terms and asked the subjects to describe in detail as much of the image containing the stimulus term as possible. The experimenter recorded a response as correct if the target object or an approximation to the target was supplied.

\section{RESULTS}

\section{Accuracy of Recall}

With respect to mean recall of response items, a $2 \times 3$ (image type $\times$ condition) analysis of variance yielded a significant main effect involving conditions $[F(2,76)=$ $\left.3.42, M S_{\mathrm{e}}=.03, p<.05\right]$. Pictorial images were recalled better than were either concealed or separate ones. Newman-Keuls pairwise comparisons showed that pictorial mean recall (5.06) was different from both concealed (3.42) and separate (3.13). The latter two conditions, however, did not differ from each other.

A main effect of image type was also found $[F(1,38)$ $\left.=10.18, M S_{\mathrm{e}}=.79, p<.01\right]$, with normal images benefiting recall more than did bizarre ones. The respective means were 4.43 and 3.57. Finally, the image type $x$ condition interaction revealed that pictorial recall was better than were concealed and separate under both normal and bizarre images $\left[F(2,76)=4.54, M S_{\mathrm{e}}=.03\right.$, $p<.05]$. Pairwise comparisons showed that the pictorial image types significantly differed from the other conditions, although not from each other (see Table 2).

\section{Rated Vividness of Images}

The three conditions also differed significantly in vividness $\left[F(2,76)=6.92, M S_{\mathrm{e}}=.65, p<.01\right]$. Pairwise comparisons fell into the same pattern as for recall. Pictorial images were rated as more vivid than either concealed or separate ones, which did not differ. Respective mean ratings were $2.72,3.45$, and 3.42. As in the previous analysis, an image-type effect was found, with bizarre images being less vivid than were normal types $[F(1,38)$ 
Table 2

Mean Number of Items Recalled as a Function of Image Type and Conditions

\begin{tabular}{lcc}
\hline & \multicolumn{2}{c}{ Image Type } \\
\cline { 2 - 3 } Conditions & Normal & Bizarre \\
\hline Pictorial & 5.13 & 4.99 \\
Concealed & 3.98 & 2.86 \\
Separate & 3.70 & 2.57 \\
\hline
\end{tabular}

Note-Maximum score for each condition $=6.00$.

Table 3

Average Vividness Ratings as a Function of Image Type and Conditions

\begin{tabular}{lcc}
\hline & \multicolumn{2}{c}{ Image Type } \\
\cline { 2 - 3 } Conditions & Normal & Bizarre \\
\hline Pictorial & 2.34 & 3.10 \\
Concealed & 2.72 & 4.18 \\
Separate & 2.90 & 3.94 \\
\hline
\end{tabular}

Note-Most vivid $=1.00$; least vivid $=7.00$.

$\left.=6.54, M S_{\mathrm{e}}=.15, p<.05\right]$. The mean bizarre rating was 3.55 , and the mean normal was 2.81 .

The type $\times$ condition interaction revealed that normal and bizarre pictorial images were rated more vivid than the corresponding concealed and separate types $[F(2,76)$ $\left.=7.51, M S_{\mathrm{e}}=.65, p<.01\right]$. As Table 3 shows, all bizarre conditions were rated as less vivid than the normal ones. The least vivid of all conditions was the bizarre concealed condition, as indicated by pairwise comparisons.

\section{DISCUSSION}

To summarize the major findings, recall of target items was poorer under concealed conditions than it was under pictorial; also, the vividness of concealed imagery was rated lower than pictorial, replicating the results of Keenan and Moore (1979) and others (e.g., Kerr \& Neisser, 1983). As previously indicated by Sheehan and Neisser (1969), strongly worded instructions to conceal particular targets could have had the effect of making the objects less visible and subsequently less vivid.

With respect to bizarre types of images, the experimental hypotheses were supported: Recall of targets was poorer and vividness ratings were lower than were those for the more normal types, confirming past research (Collyer, Jonides, \& Bevan, 1972; Hauck, Walsh, \& Kroll, 1976; Nappe \& Wollen, 1973; Wollen \& Cox, 1981). Interactions revealed a similar conditions pattern occurring with both types, with the exception that normal images were still more effective than were bizarre images. Specific results from these interactions showed that bizarre and normal pictorial images elicited the best recall, while bizarre concealed ones were rated the least vivid of all conditions. It would appear that both bizarre and concealed imagery were adversely affected by task demands involving strongly worded instructions.

On the basis of our results, we propose that bizarre, concealed mental layouts are basically ineffective mnemonic aids on immediate recall tests. Further research is required to determine whether these images can be effective over extended retention intervals to attain a better understanding of which types of images benefit recall performance and which characteristics of these layouts are the most relevant ones for information-processing purposes.

\section{REFERENCES}

Cox, S. D., \& Wollen, K. A. (1981). Bizarreness and recall. Bulletin of the Psychonomic Society, 18, 244-245.

Collyer, S. C., Jonides, J., \& BEVAN, W. (1972). Images as memory aids: Is bizarreness helpful? American Journal of Psychology, 85, 31-38.

Hauck, P. D., Walsh, C. C., \& Kroll, N. E. A. (1976). Visual imagery mnemonics: Common vs. bizarre mental images. Bulletin of the Psychonomic Society, 7, 160-162.

IACCINO, J. F., \& SPIREK, P. (1988). Long-term retention of plausible vs. bizarre paired associates as a function of cued recall. Perceptual \& Motor Skills, 67, 531-537.

KeEnAN, J. M. (1983). Qualifications and clarifications of images of concealed objects: A reply to Kerr and Neisser. Journal of Experimental Psychology: Learning, Memory, \& Cognition, 9, 222-230.

KeEnAN, J. M., \& MoORE, R. E. (1979). Memory for images of concealed objects: A reexamination of Neisser and Kerr. Journal of Experimental Psychology: Human Learning \& Memory, 5, 374-385.

KeRR, N. H., \& NeISSER, U. (1983). Mental images of concealed objects: New evidence. Journal of Experimental Psychology: Learning, Memory, \& Cognition, 9, 212-221.

KozMINSKY, E. (1981). Retrieving concealed objects. Unpublished manuscript, Department of Education, Ben Gurion University of the Negev, Beer Sheva, Israel.

Kroll, N. E., Schepeler, E. M., \& ANGin, K. T. (1986). Bizarre imagery: The misremembered mnemonic. Journal of Experimental Psychology: Learning, Memory, \& Cognition, 12, 42-53.

NAPPE, G. W., \& Wollen, K. A. (1973). Effects of instructions to form common and bizarre mental images on retention. Journal of $E x$ perimental Psychology, 100, 6-8.

NeISSER, U., \& KeRR, N. H. (1973). Spatial and mnemonic properties of visual images. Cognitive Psychology, 5, 138-150.

Paivio, A., Yuille, J. C., \& Madigan, S. A. (1968). Concreteness, imagery and meaningfulness values for 925 nouns. Journal of Experimental Psychology Monograph Supplement, 76(1, Pt. 2).

SENTER, R. J., \& HofFMAN, R. R. (1976). Bizarreness as a nonessential variable in mnemonic imagery: A confirmation. Bulletin of the Psychonomic Society, 7, 163-164.

SheEHAN, P. W. (1967). A shortened form of Bett's questionnaire upon mental imagery. Journal of Clinical Psychology, 23, 386-389.

Sheehan, P. W., \& Neisser, U. (1969). Some variables affecting the vividness of imagery in recall. British Journal of Psychology, 60 , 71-80.

Wollen, K. A., \& Cox, S. (1981). Sentence cuing and the effectiveness of bizarre imagery. Journal of Experimental Psychology: Human Learning \& Memory, 7, 386-392.

Wollen, K. A., Weber, A., \& LoWry, D. H. (1972). Bizarreness versus interaction of mental images as determinants of learning. Cognitive Psychology, 3, 518-523.

Wood, G. (1967). Mnemonic systems in recall. Journal of Educational Psychology Monographs, 58(6, Whole No. 645).

Zimler, J., \& KeENAN, J. M. (1983). Imagery in the congenitally blind: How visual are visual images? Journal of Experimental Psychology: Learning, Memory, \& Cognition, 9, 269-282.

(Manuscript received February 13, 1989.) 